\title{
Mutation and Linkage Analysis in Genetic Counseling for Phenylketonuria in Kuwait
}

\author{
Elena Samilchuk Ibrahim Al-Suliman Gadeer Naqi \\ Sadika Al-Awadi \\ Kuwait Medical Genetics Center, Kuwait
}

\section{Key Words}

Phenylketonuria PAH gene - Mutation .

Linkage analysis · IVS10nt546 · Kuwait

\begin{abstract}
Objective: Phenylketonuria (PKU) is caused by mutations in the PAH gene. More than 250 PAH mutations have been identified, and their distribution among different populations varies significantly. Though PKU is prevalent in Kuwait, the molecular bases of the underlying genetic variants have never been studied. Methods: DNA from 20 PKU patients belonging to 11 families of Arabic origin as well as 30 healthy relatives was extracted using a 'salting-out' method. DNA samples were analyzed by polymerase chain reaction (PCR)/restriction fragment length polymorphism (RFLP) tests for 5 common PAH mutations (IVS10nt546, R2610, IVS12nt1, IVS2nt1, $\mathrm{R} 408 \mathrm{~W})$. Linkage analysis and PAH haplotyping was carried out by the study of variable number tandem repeat and Bgll, Pvull-a, Pvull-b, Mspl, Xmnl polymorphism by PCR or
\end{abstract}

\begin{tabular}{ll}
\hline KARGER & ( 1999 S. Karger AG, Basel \\
Fax +4161306 1234 34 & \\
$\begin{array}{l}\text { E-Mail karger@karger.ch } \\
\text { www.karger.com }\end{array}$ & $\begin{array}{l}\text { Accessible online at: } \\
\text { http://BioMedNet.com/karger }\end{array}$
\end{tabular}

PCR/RFLP tests. Results: Seven patients from 4 families were found to be homozygous for IVS10nt546, thus proving the frequency of this mutation among PKU chromosomes in Kuwait to be $36.4 \%$. The four other PAH mutations were absent among the patients studied. The linkage analysis was informative in all but 1 of the families. Seven PAH haplotypes were identified among 22 PKU chromosomes from Kuwait. All the IVS10nt546 chromosomes had identical haplotype.

\section{Introduction}

Phenylketonuria (PKU) is one of the commonest metabolic disorders in many populations including Kuwait [1, 2]. A pilot screening program carried out in Kuwait in 19861987, estimated the incidence of PKU as 1 in 6,479 newborns [3]. However, the molecular basis of the underlying genetic variants has never been studied. Meanwhile, such analysis is extremely important for genetic counseling.

Dr. Elena Samilchuk

Kuwait Medical Genetics Center, PO Box 31121

Sulaibikhat 80901 (Kuwait)

Tel./Fax +9655735201 
Table 1. PCR/RFLP tests for PAH mutations used in the study

\begin{tabular}{lllll}
\hline Mutation & $\begin{array}{l}\text { Restriction } \\
\text { enzyme }\end{array}$ & $\begin{array}{l}\text { PCR } \\
\text { product } \\
\text { bp }\end{array}$ & $\begin{array}{l}\text { Digest } \\
\text { wild-type } \\
\text { bp }\end{array}$ & $\begin{array}{l}\text { Digest } \\
\text { mutant } \\
\text { bp }\end{array}$ \\
\hline IVS10nt546 & DdeI & 295 & 295 & $246+49$ \\
R261Q & HinfI & 291 & $136+155$ & 291 \\
IVS12nt1 & RsaI & 156 & $133+23$ & 156 \\
IVS2nt1 & MnlI & 134 & $82+52$ & 134 \\
R408W & MnlI & 232 & $126+106$ & 232 \\
\hline
\end{tabular}

Molecular tests allow not only detection of affected individuals, but also of normal carriers who cannot be identified by the biochemical methods used for the diagnosis of PKU.

PKU is caused mainly by mutations in the PAH gene coding for phenylalanine hydroxylase. More than $250 \mathrm{PAH}$ mutations are known [4]. Only a few are common, at least in some populations, while the majority of the PAH mutations are rare. The distribution of the PAH mutations among different populations varies significantly. In Europe, $4 \mathrm{PAH}$ mutations, namely IVS10nt546, R261Q, IVS12nt 1 and R408W are the most frequent [5]. Of special interest among them is IVS10nt546, which was found at high frequency among PKU chromosomes from the Mediterranean and neighboring regions, and therefore could be expected to be present in Kuwait [5-8]. Another mutation promising for testing in Arabic populations is IVS2nt2, which was reported to be quite common among Palestinian Arabs [9].

For the purpose of genetic counseling, the lack of information on the PAH mutations can be partly compensated for by linkage analysis using polymorphic markers inside the $\mathrm{PAH}$ gene and in the flanking regions.

Polymerase chain reaction (PCR) tests designed for detection of several restriction fragment length polymorphisms (RFLPs) and variable number tandem repeat (VNTR) at the PAH locus greatly facilitated the use of linkage analysis in PKU families [10-14].

We present here the results of the mutation and linkage analysis in PKU families from Kuwait.

\section{Materials and Methods}

Twenty PKU patients from 11 families of Arab origin as well as 30 healthy family members were studied. In total, 22 PKU chromosomes are accounted for. All families except 1 were consanguineous. DNA was extracted from the peripheral blood using a 'saltingout' technique [15].

PCR was performed in the Perkin Elmer 9600 system. The PCR mixture contained $10 \mathrm{~m} M$ Tris- $\mathrm{HCl}$, pH 8.3, $50 \mathrm{~m} M \mathrm{KCl}, 1.5 \mathrm{~m} M \mathrm{MgCl}_{2}, 0.001 \%(\mathrm{~m} / \mathrm{w})$ gelatine, $50 \mu M$ of each dNTP, $0.4 \mu M$ of the primers and 0.75 units of Taq polymerase (Perkin Elmer, Branchville, N.J., USA) in a total volume of $25 \mu 1$.

\section{Direct Tests for PAH Mutations}

Direct testing for 5 common PAH mutations (IVS10nt546, R261Q, IVS12nt1, IVS2nt1, R408W) was performed by PCR amplification of PAH gene fragments encompassing the mutation sites followed by digestion with restriction enzymes (table 1). The primers for the R261Q and IVS12nt1 mutations were kindly given by Dr. G. Besley (Willink Biochemical Genetics Unit, Manchester, UK). The primers for IVS10nt546 and R408W have been reported previously $[6,8]$. The sequence of the flanking regions of exon 2 was kindly provided by Dr. P. Nowacki (McGill University, Montreal, Canada). Primers for detection of IVS2nt 1 (5'-aagacaactgcaatcaaaat- $3^{\prime}$ and 5'-gctacgacattatccaagac- $3^{\prime}$ ) were designed with computer program 'Oligo 5.0' (National Biosciences Inc., Plymouth, USA).

\section{Linkage Analysis and PAH Haplotyping}

Five RFLPs $(B g / \mathrm{I}, P v u \mathrm{II}-\mathrm{a}, P v u \mathrm{II}-\mathrm{b}, M s p \mathrm{I}, X m n \mathrm{I})$ and VNTR were analyzed. The sequences of PCR primers and characteristics of PCR test for analysis of these markers have been reported elsewhere [10-14]. PCR products were digested by restriction enzymes $B g l \mathrm{I}, P v u \mathrm{II}, M s p \mathrm{I}, X m n \mathrm{I}$ according to the manufacturer's protocols and further analyzed by agarose gel electrophoresis. The sizing of VNTR was performed by agarose or polyacrylamide gel electrophoresis. 


\section{Results}

All PKU patients were tested for $5 \mathrm{PAH}$ mutations (IVS10nt546, R261Q, IVS12nt1, IVS2nt1, R408W). Seven patients from 4 families were found to be homozygous for IVS10nt546, thus proving the frequency of this mutation among PKU chromosomes in Kuwait to be $36.4 \%$. The test for IVS10nt546 carried out in healthy members of these families detected the carriers. In addition to the parents (obligatory carriers), several healthy siblings were also found to be heterozygotes for IVS10nt546 (fig. 1). Four other PAH mutations (R261Q, IVS12nt1, IVS2nt1, R408W) were absent in Kuwaiti PKU patients.

Linkage analysis using VNTR, $B g l \mathrm{I}, P v u \mathrm{II}-$ a, PvuII-b, MspI and $X m n I$ polymorphisms was carried out in 5 of the 7 PKU families for which the PAH mutation remains unknown. The other 2 families were not examined because DNA samples from the parents were not available. This analysis was informative in all but 1 of the families. If more than 1 marker was informative, the results were concordant. Using this approach, several normal carriers were identified among healthy siblings. The representative results of linkage analysis are shown in figure 2.

Analysis of RFLPs and VNTR patterns in all 22 PKU chromosomes from Kuwait revealed 7 different PAH haplotypes (table 2). The IVS10nt546 chromosomes from all 4 families in which this mutation was found had identical haplotype. The patients from 6 other consanguineous families (PAH mutation unknown) who were homozygous for VNTR and RFLPs alleles revealed $5 \mathrm{PAH}$ haplotypes. One of these haplotypes was also identified on a paternal PKU chromosome from the single nonconsanguineous family. However, the maternal haplotype in this family was different from all others observed in Kuwait.

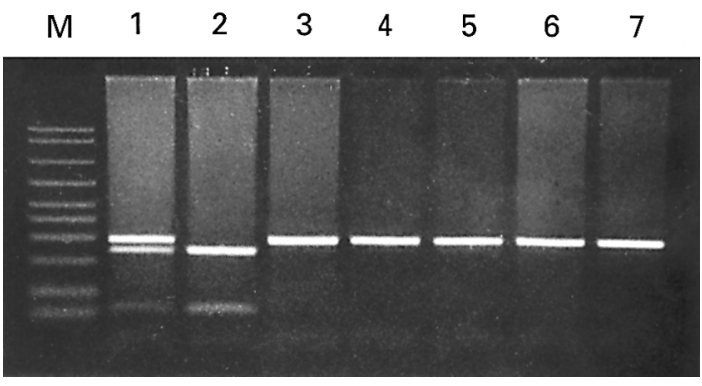

Fig. 1. $P C R / R F L P$ detection of the IVS10nt546 mutation. $\mathrm{M}=$ Molecular weight markers: 50, 100, 200, $300 \mathrm{bp}$ etc; 1 = heterozygote for IVS10nt546; 2 = homozygote for IVS10nt546; 3-7 = negative for IVS10nt546.

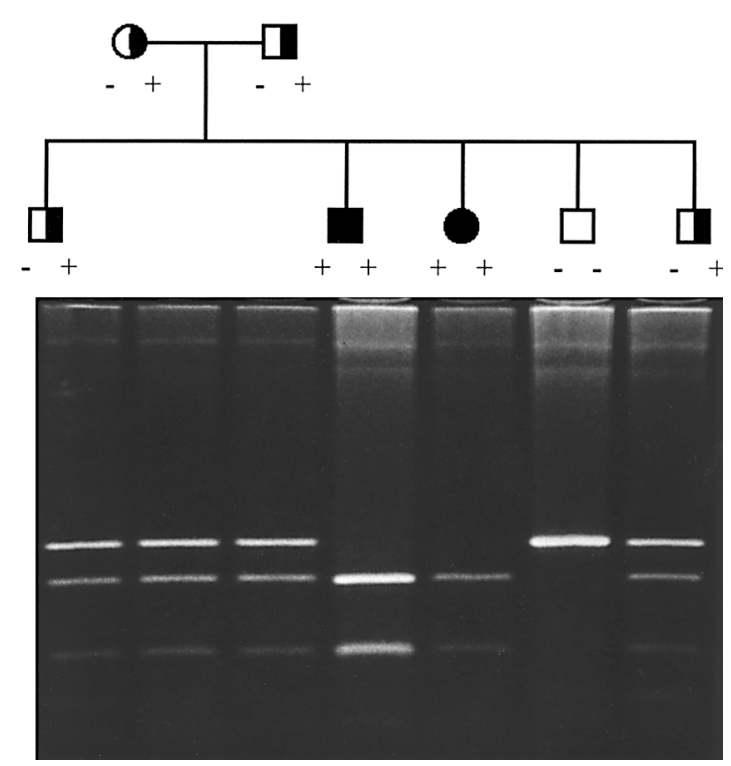

Fig. 2. Linkage analysis using $M s p I$ polymorphism. Affected children are homozygous $M s p I+/+$. It means that both maternal and paternal PKU chromosomes carry the $M s p I+$ allele, while the normal chromosomes are $M s p \mathrm{I}-$. Therefore, 2 healthy siblings $M s p \mathrm{I}+/-$ are carriers for PKU mutation. The third healthy sibling MspI -/- is negative for PKU mutation. 
Table 2. PAH haplotypes of PKU chromosomes from Kuwait

\begin{tabular}{lllllllll}
\hline Mutation & \multicolumn{2}{l}{$\begin{array}{l}\text { Chromo- } \\
\text { somes }\end{array}$} & & & & & & \\
& & & & & & \\
\hline IVS10nt5 546 & 8 & + & - & + & + & - & 7 \\
Unknown & 2 & - & + & - & + & - & 7 \\
Unknown & 2 & - & + & - & + & - & 8 \\
Unknown & 4 & + & - & + & + & - & 8 \\
Unknown & 3 & + & - & + & + & - & 9 \\
Unknown & 2 & - & + & - & + & - & 3 \\
Unknown & 1 & & + & - & - & - & + & 8 \\
\hline
\end{tabular}

'+' or '-': presence or absence of restriction site.

1 ' $3,7,8,9$ ': number of repeated units in VNTR.

\section{Discussion}

Our study provides the first data on $\mathrm{PAH}$ mutations and haplotypes among PKU chromosomes in Kuwait. The investigated samples represent practically all identified PKU chromosomes in Kuwait. Though several PKU patients were not available for this study, they were siblings of the probands studied and therefore had the same PKU chromosomes.

The IVS10nt546 mutation was found to be quite frequent in Kuwait $36.4 \%$ of PKU chromosomes). The identification of this mutation in Kuwait is not surprising, as it is quite common in the Mediterranean area. A similar result was also observed for glucose-6-phosphate dehydrogenase deficiency: the 563 $\mathrm{C} \rightarrow \mathrm{T}$ mutation of the G6PD gene common in the Mediterranean region was also found to be very frequent in Kuwait [16]. The majority of IVS10nt546 chromosomes found in different populations have a common PAH haplotype, thus suggesting the common origin of this mutation [4]. Most probably IVS10nt546 arose in Turkey from where it spread throughout the Mediterranean and neighboring regions [17]. The PAH haplotype of IVS10nt546 chromosomes from Kuwait can be provisionally attributed to this common haplotype; however, two additional RFLPs should be analyzed to reach the final conclusion.

Unfortunately, these two RFLPs can be detected only by Southern blotting, which restricts the practical use of these markers.

The PAH haplotypes in all consanguineous families were identical on both maternal and paternal PKU chromosomes, thus suggesting an identical PAH mutation (homozygosity by descent). Indeed, in all 4 families with known mutation (IVS10nt546), the probands were homozygotes for this mutation. In contrast, 2 different PAH haplotypes were revealed in the single nonconsanguineous family. Thus, different mutations might be expected on maternal and paternal PKU chromosomes in this case.

It can be suggested that different mutations are present on 7 PAH haplotypes identified among Kuwaiti PKU chromosomes. It is interesting that one of these haplotypes (allele 3 for VNTR; allele 'plus' for $P v u \mathrm{II}-\mathrm{a}$ and $M s p \mathrm{I}$; allele 'minus' for $B g / \mathrm{I}$ and $\mathrm{XmnI}$ ) is often associated with the R408W mutation. However, in contrast to expectation, R408W was not found in the Kuwaiti patient with this haplotype. 
Linkage analysis can be used successfully in most PKU families from Kuwait. Indeed, several carriers have been identified by this approach in PKU families with unknown mutation. However, linkage analysis has some limitations. Unfortunately, it is virtually useless in premarital genetic counseling which is more acceptable in Kuwait than prenatal diagnosis. Thus, the identification of the PAH mutations in all PKU probands from Kuwait is warranted. This can be achieved by analysis of the whole coding region and all splicing sites of the PAH gene. A search for IVS10nt546 would be of use in premarital genetic counseling taking into consideration the high PKU incidence as well as the high frequency of IVSnt546 in Kuwait.

\section{Acknowledgments}

The authors are thankful to Dr. Girish Yadav and Dr. Nawal Al Qaoud for providing information on PKU cases in Kuwait.

\section{References}

1 Scriver CR, Kaufman S, Eisensmith RC, Woo SLC: The hyperphenylalaninemias; in Scriver CR, Beuadet AL, Sly WS, Valle D (eds): The Metabolic and Molecular Bases of Inherited Diseases. New York, McGrawHill, 1995, pp 1015-1075.

2 Yadav GC, Reavey PC: Aminoacidopathies: A review of 3 years experience of investigations in a Kuwait hospital. J Inherited Metab Dis 1988;11:277-284.

3 Tebbi AS: Autosomal recessive disorders among Arabs: An overview from Kuwait. J Med Genet 1994;31: 224-233.

4 Hoang L, Byck S, Prevost L, Scriver CR: PAH Mutation Analysis Consortium Database: A database for disease-producing and other allelic variation at the human PAH locus. Nucleic Acids Res 1996;24:127131.

5 Eisensmith RC, Okano Y, Dasovich M, Wang T, Guttler F, Lou H, Guldberg P, Lichter-Konecki U, Konecki DS, Svensson E, Hagenfeldt L, Rey F, Munnich A, Lyonnet S, Cockburn F, Connor JM, Pembrey ME, Smith I, Gitzelmann R, Steinmann B, Apold J, Eiken HG, Giovannini M, Riva E, Longhi R, Romano C, Cerone $\mathrm{R}$, Naughten ER, Mullins C, Cahalane S, Ozalp I, Fekete G, Schuler D, Berencsi GY, Nasz I, Brdicka R, Kamaryt J, Pijackova A, Cabalska B, Boszkowa K, Schwartz E, Kalinin VN, Jin L, Chakraborty
R, Woo SLC: Multiple origins for phenylketonuria in Europe. Am J Hum Genet 1992;52:1355-1365.

6 Ozguk M, Ozalp I, Coskun T, Yilmaz E, Erdem H, Ayter S: Mutation analysis in Turkish phenylketonuria patients. J Med Genet 1993;30:129130.

7 Goltsov A, Kouzmine A, Eisensmith RC, Effat L, Temtamy S, Rushdi S, Abdel-Meguid N, Woo SLC: Molecular basis of phenylketonuria in Egypt. Am J Hum Genet 1994; 55(suppl):A221.

8 Guldberg P, Romano V, Ceratto N, Bosco P, Ciuna M, Indelicato A, Mollica F, Meli C, Giovannini M, Riva E, Biasucci G, Henriksen KF, Guttler F: Mutational spectrum of phenylalanine hydroxylase deficiency in Sicily: Implications for diagnosis of hyperphenylalaninemia in southern Europe. Hum Mol Genet 1993;2:1703-1707.

9 Kleiman S, Bernstein J, Schwartz G, Eisensmith RC, Woo SLC: A defective splice site at the phenylalanine hydroxylase gene in phenylketonuria and benign hyperphenylalaninemia among Palestinian Arabs. Hum Mutat 1992;1:340-343.

10 Goltsov AA, Eisensmith RC, Konecki DS, Lichter-Konecki U: Association between mutations and a VNTR in the human phenylalanine hydroxylase gene. Am J Hum Genet 1992;51:627-636.
11 Dworniczak B, Wedemeyer N, Eigel A, Horst J: PCR detection of the $P v u$ II (Ea) RFLP at the human phenylalanine hydroxylase (PAH) locus. Nucleic Acids Res 1991;19: 1958.

12 Dworniczak B, Wedemeyer N, Horst J: PCR detection of the $B g / \mathrm{II}$ RFLP at the human phenylalanine hydroxylase (PAH) locus. Nucleic Acids Res 1991;19:1958.

13 Wedemeyer N, Dworniczak B, Horst J: PCR detection of the MspI (Aa) RFLP at the human phenylalanine hydroxylase (PAH) locus. Nucleic Acids Res 1991;19:1959.

14 Goltsov AA, Eisensmith RC, Woo SL: Detection of the XmnI RFLP at the human PAH locus by PCR. Nucleic Acids Res 1991;20:927.

15 Miller SA, Dykes DD, Polesky HF: A simple salting out procedure for extracting DNA from human nucleated cells. Nucleic Acids Res 1988;16:1215.

16 Samilchuk E, D’Souza B, Al-Awadi S: Population study of common G6PD mutations in Kuwait. Hum Heredity 1999;49:41-44.

17 Eisensmith RC, Woo SLC: Population genetics of phenylketonuria. Acta Paediatr 1994;407(suppl):1926. 